\title{
Epithelial cell culture models for the prevention and therapy of clinical breast cancer (Review)
}

\author{
NITIN TELANG ${ }^{1}$ and MEENA KATDARE ${ }^{2}$ \\ ${ }^{1}$ Cancer Prevention Research Program, Palindrome Liaisons, Montvale, NJ; \\ ${ }^{2}$ Skin of Color Research Institute, Hampton University, Hampton, VA, USA
}

Received October 1, 2011; Accepted December 20, 2011

DOI: $10.3892 / \mathrm{ol} .2012 .561$

\begin{abstract}
Clinical breast cancer progresses via a multi-step carcinogenic process wherein genetic, molecular, endocrine and dietary factors play significant roles in the pathogenesis, prevention and therapy of the disease. Preclinical cell culture models, expressing clinically relevant genetic and endocrine defects and exhibiting quantifiable cancer risk, may provide facile, clinically translatable approaches to identify molecular targets and susceptible mechanistic pathways for the efficacy of novel interventional approaches. This review summarizes laboratory investigations focused on i) developing murine and human mammary tissue-derived cell culture models; ii) optimizing mechanism-based quantitative endpoint biomarker assays specific for carcinogenic risk and preventive/ therapeutic efficacy; and iii) providing quantifiable proof-ofprinciple evidence for validation of the present cell culture approaches, capable of prioritizing efficacious lead compounds for subsequent in vivo animal studies and clinical trials for the prevention/therapy of breast cancer. Epithelial cell culture models are developed and characterized where the carcinogenic process is initiated by the targeted expression of clinically relevant oncogenes. The cell culture systems from mouse mammary tissue are in vitro approaches that complement the Ras and Myc transgenic mouse models. The human mammary tissue-derived systems are in vitro models for chemoendocrine, therapy-resistant, clinical, pre-invasive ER-/PR-/HER-2+ comedo ductal carcinoma in situ, $\mathrm{ER}^{+} / \mathrm{PR}^{+}$chemoendocrine therapy-responsive breast cancer and ER-/PR-/HER-2- triplenegative chemoendocrine, therapy-resistant breast cancer. The oncogene-initiated phenotypes exhibit loss of homeostatic
\end{abstract}

Correspondence to: Dr Nitin Telang, Cancer Prevention Research Program, Palindrome Liaisons, 10 Rolling Ridge Road, Suite B, Montvale, NJ 07645-1559, USA

E-mail: entitytoo@gmail.com

Dr Meena Katdare, Skin of Color Research Institute, Hampton University, PO Box 6035, Hampton, 23668 VA, USA

E-mail: meena.katdare@hamptonu.edu

Key words: epithelial cell culture, breast carcinogenesis, prevention/ therapy growth control, downregulation of cell apoptosis and gain of carcinogenic risk in vitro, as well as transplantable tumor development in vivo. Numerous mechanistically distinct, synthetic pharmacological agents, as well as naturally occurring dietary compounds, re-establish homeostatic growth control via cell cycle arrest and/or induction of cell apoptosis, downregulate oncogene-mediated cell signaling pathways, modulate the expression of numerous cell cycle regulatory and apoptosisspecific proteins and reduce carcinogenic risk in pre-neoplastic and carcinoma-derived cell culture models. These data validate the present cell culture approaches as novel, mechanism-based screens to evaluate and prioritize promising lead compounds for the prevention/therapy of clinical breast cancer.

\section{Contents}

1. Introduction

2. Cell culture models and mechanistic biomarkers

3. Status of biomarker expression in cell culture models

4. Response to chemo-preventive agents

5. Mechanisms for efficacy of chemo-preventive agents

6. Conclusions and future directions

\section{Introduction}

Breast cancer remains a prevalent cause of mortality in the female US population. The American Cancer Society estimates a projected 230,480 new cases of invasive breast cancer, and 39,520 cancer-related mortalities in 2012 (1). Genetic background, hormones and diet are major risk factors for the multi-step carcinogenic process of the disease (2). A total of $75-80 \%$ of breast cancers are diagnosed as hormone responsive and exhibit acceptable response to endocrine-targeted therapy that utilizes selective estrogen receptor (ER) modulators, aromatase inhibitors or progesterone receptor (PR)-targeted anti-progestins with or without chemotherapy $(3,4)$. By contrast, $7-15 \%$ of breast cancers do not express hormone and/or growth factor receptors (ER $/ \mathrm{PR}^{-} / \mathrm{HER}-2^{+}$or $\left.\mathrm{ER}^{-} / \mathrm{PR}^{-} / \mathrm{HER}-2^{-}\right)$, and therefore may exhibit only a modest response to conventional chemo-endocrine therapy. These cancer subtypes present a challenge to currently available treatment options (5-8). Long-term conventional chemo-endocrine therapy is frequently 
Table I. Epithelial cell culture models for mouse breast cancer.

\begin{tabular}{|c|c|c|}
\hline Cell line & Origin & Model \\
\hline C57MG & $\mathrm{C} 57 \mathrm{Bl} / 6 \mathrm{~J}$ & Normal mammary gland \\
\hline C57MG/DMBA & DMBA transformed & Carcinogen (pre-neoplastic) \\
\hline $\mathrm{C} 57^{\text {Ras }}$ & Ras transfectant & Ras oncogene (pre-neoplastic) \\
\hline MMEC & $\mathrm{BALB} / \mathrm{c}$ & Normal mammary gland \\
\hline MMEC-Ras & Ras transfectant & Ras oncogene (pre-neoplastic) \\
\hline $\mathrm{T}_{1}-\mathrm{Pr}_{1}$ & Ras AI colony & Primary tumor \\
\hline MMEC-Myc & Myc transfectant & Myc oncogene (Pre-neoplastic) \\
\hline $\mathrm{Myc}_{3}-\operatorname{Pr}_{1}$ & Myc AI colony & Primary tumor \\
\hline
\end{tabular}

DMBA, 7, 12-dimethylbenz (a) anthracene; AI, anchorage-independent.

associated with acquired tumor resistance and other adverse systemic toxicity, compromising patient compliance $(3,4,6,9)$.

The global gene expression profile-based molecular classification of clinical breast cancer facilitates better prediction of disease progression and chemotherapeutic response, thereby enhancing the sensitivity and specificity of targeted therapeutic intervention (5-9). The chemo-endocrine therapy-resistant breast cancer, which expresses the HER-2 oncogene, is responsive to the HER-2-specific antibody and/ or small molecule inhibitors of HER-2 action. In addition, HER-2-specific therapy is noted to enhance the tumor response to conventional chemotherapy (10-13). Triple-negative breast cancer (TNBC) lacks the expression of the ER, PR and of the HER-2 oncogene. This cancer subtype exhibits an acceptable response only to conventional chemotherapy $(5-8,14)$. These intrinsic limitations of conventional chemo-endocrine therapy have resulted in numerous investigations in order to examine the efficacy of natural phytochemicals and herbal medicinal preparations. Unlike synthetic chemotherapeutic drugs, the naturally occurring compounds commonly exhibit favorable toxicity profiles and may effectively interact with conventional chemo-endocrine therapeutic agents to enhance their efficacy and reduce therapy-associated toxicity (15-19).

As a complementary approach to conventional in vitro and in vivo preclinical cell culture models, considerable efforts have focused on developing reliable mammary epithelial cell culture models that express clinically relevant genetic defects and exhibit a quantifiable risk for carcinogenesis $(20,21)$. Similar models derived from human mammary tissue are unique in providing a high potential for clinical translatability $(8,20,21)$. These experimental approaches complement genetically predisposed animal models and immune compromised xeno-transplant models and, thereby, facilitate a mechanism-based evaluation of the preventive/therapeutic efficacy of lead compounds and their prioritization for subsequent in vivo preclinical studies for future clinical trials.

The long-term overall objective of our research directions have focused on testing the hypotheses that i) oncogene initiated multi-step carcinogenesis is associated with the loss of homeostatic growth control and gain of carcinogenic risk; ii) mechanistically distinct chemo-preventive agents effectively reverse the early occurring events in the carcinogenic process via restoring the loss of homeostatic growth control and reducing the gain of carcinogenic risk; and iii) human mammary carcinoma-derived cell culture models that are characterized for the status of homeostatic growth control and whose tumorigenic potential provide mechanism-based approaches to identify susceptible molecular pathways and novel molecular targets for the therapeutic efficacy of new lead compounds. In an effort to accomplish the overall objectives, we developed novel mammary epithelial cell culture models for chemo-endocrine therapy responsive and resistant clinical breast cancer. These models have been characterized using several mechanistic end-point biomarker assays, and have been validated as short-term screening approaches for the efficacy of potential preventive/therapeutic lead compounds for clinical breast cancer.

The present review summarizes the data generated from previously published, as well as recently completed studies, provides an overview of the potential applications of the developed models and discusses future directions.

\section{Cell culture models and mechanistic biomarkers}

Stable transfection and resultant overexpression of clinically relevant Ras, Myc and HER-2 oncogenes in immortalized, but non-tumorigenic, mammary epithelial cell lines confers tumorigenic transformation in the target cells $(20,21)$.

The data shown in Table I describe cell culture models developed from immortalized mouse mammary epithelial cells. The chemical carcinogen 7, 12-dimethylbenz (a) anthracene (DMBA), as well as Ras and Myc oncogenes, independently induce the multi-step carcinogenic process. These initiated cells exhibit anchorage-independent colony formation in vitro and tumorigenic potential upon in vivo transplantation. The primary tumors derived from the pre-neoplasically transformed cells exhibit a persistent expression of the biomarkers for carcinogenic risk (22-24).

The data shown in Table II describe the developed models from human mammary tissue. The human mammary epithelial cell line developed from the mammary terminal duct-lobular unit (TDLU), exhibits the induction of a multi-step carcinogenic process in response to either chemical carcinogens, benzo(a)pyrene (BP) or DMBA, or to transfection with the HER-2 oncogene $(20,25)$. These models exhibit the expression of end-point biomarkers. The human breast carcinoma-derived 
Table II. Epithelial cell culture models for human breast cancer.

\begin{tabular}{lll}
\hline Cell line & \multicolumn{1}{c}{ Origin } & \multicolumn{1}{c}{ Model } \\
\hline 184-B5 & TDLU & Normal breast \\
184-B5/BP & BP transformed & Carcinogen (pre-neoplastic) \\
184-B5/DMBA & DMBA transformed & Carcinogen (pre-neoplastic) \\
$184-B 5 / H E R$ & HER-2 transfectant & HER-2 oncogene (pre-neoplastic) \\
MCF-7 & Breast carcinoma & ER $^{+} / \mathrm{PR}^{+} / \mathrm{HER}-2^{-}$endocrine therapy-responsive breast cancer \\
MDA-MB-231 & Breast carcinoma & ER $^{-} / \mathrm{PR}^{-} / \mathrm{HER}-2^{-}$chemo-endocrine therapy-resistant, triple-negative breast cancer
\end{tabular}

TDLU, terminal duct-lobular unit; BP, benzo(a)pyrene; DMBA, 7, 12-dimethylbenz (a) anthracene.

Table III. Mechanistic biomarkers.

\begin{tabular}{|c|c|c|}
\hline Biomarker & Assay & Endpoint \\
\hline \multirow{5}{*}{$\begin{array}{l}\text { Homeostatic } \\
\text { growth control }\end{array}$} & Saturation density & Cell viability \\
\hline & Population doubling time & Cell replication \\
\hline & Cell cycle progression & $\mathrm{G}_{1}: \mathrm{S}+\mathrm{G}_{2} / \mathrm{M}$ ratio \\
\hline & Cell apoptosis & $\% \operatorname{sub} \mathrm{G}_{0}$ \\
\hline & & $\mathrm{S}+\mathrm{G}_{2} / \mathrm{M}: \mathrm{sub} \mathrm{G}_{0}$ ratio \\
\hline \multirow[t]{2}{*}{ Carcinogenic risk } & AI growth & AI colony number \\
\hline & Tumor development & $\begin{array}{l}\text { Tumor incidence/latency of } \\
\text { transplanted cells }\end{array}$ \\
\hline \multirow[t]{2}{*}{ Molecular targets } & Cell epifluorescence & HER-2, PY-20, cyclinD1, COX-2, \\
\hline & (FACS flow cytometry) & RAR- $\beta$, Bcl- 2, Bax \\
\hline
\end{tabular}

AI, anchorage-independent; FACS, fluorescence-assisted cell sorting.

cell culture models for chemo-endocrine therapy-responsive and therapy-resistant clinical breast cancer also exhibit aberrant cell cycle progression and the upregulation of anchorage independent (AI) colony formation in vitro, and persistent tumorigenic potential in vivo.

The mechanistic endpoint biomarker assays optimized for use in the cell culture models are shown in Table III. These biomarker assays are specific and sensitive for the status of cell cycle progression, cell apoptosis, carcinogenic risk and molecular targets for the efficacy of numerous prototypical chemo-preventive/therapeutic agents.

\section{Status of biomarker expression in cell culture models}

The data generated from a comparative study on parental, Ras- and Myc-transfected mouse mammary epithelial cells are shown in Table IV. Relative to the parental MMEC cells, the preneoplastically transformed MMEC-Ras cells exhibited a $31.7 \%$ decrease in population doubling time and a 4.1-fold increase in the $\mathrm{S}+\mathrm{G}_{2} / \mathrm{M}$ : subG $\mathrm{G}_{0}$ ratio, indicating aberrant proliferation and a loss of homeostatic growth control. Furthermore, these transformed cells exhibited a 53.4-fold increase in the number of AI colonies in vitro and a high incidence of aggressive tumors upon in vivo transplantation. Similarly, the MMEC-Myc cells exhibited a $23.7 \%$ decrease, a 4 .4-fold increase and a 55 -fold increase in these biomarkers, and identical increases in the tumorigenic potential. These data are consistent with our previously published results $(21,23,24)$.

The data presented in Table $\mathrm{V}$ show a comparison of the status of biomarkers in the parental human mammary epithelial 184-B5 and the HER-2 oncogene-transfected 184-B5/ HER cells. Relative to the parental 184-B5 cells, the HER-2 oncogene-transformed 184-B5/HER cells exhibited a $55.9 \%$ decrease in the population doubling time, a 5.7-fold increase in the $\mathrm{S}+\mathrm{G}_{2} / \mathrm{M}$ : subG $\mathrm{G}_{0}$ ratio, indicating aberrant proliferation and loss of homeostatic growth control. In addition, the oncogenetransfected cells also exhibited a $75.7 \%$ increase in the number of AI colonies and a high incidence of aggressive tumors at the transplant site. These data are consistent with our previously published results $(20,25)$.

Table VI shows the data generated from the comparative study on human mammary carcinoma-derived cell culture models. In comparison to the non-tumorigenic 184-B5 cells, the $\mathrm{ER}^{+} / \mathrm{PR}^{+} / \mathrm{HER}-2^{-} \mathrm{MCF}-7$ carcinoma cells exhibited a $52.9 \%$ decrease in the population doubling time and a 5-fold increase in the $\mathrm{S}+\mathrm{G}_{2} / \mathrm{M}: \mathrm{subG}_{0}$ ratio. These cells also exhibited a 102-fold increase in the number of AI colonies and a high incidence of aggressive tumors at the transplant site. These data are consistent with our previous studies $(25,26)$. Due to their specific hormone receptor and growth factor expression status, these cells represent a preclinical cell culture model for chemoendocrine therapy-responsive clinical breast cancer. The data 
Table IV. Aberrant proliferation and carcinogenic risk in oncogene-transfected mouse mammary epithelial cells.

\begin{tabular}{|c|c|c|c|}
\hline \multirow[t]{2}{*}{ Biomarker } & \multicolumn{3}{|c|}{ Cell line } \\
\hline & MMEC & MMEC-Ras & MMEC-Myc \\
\hline \multicolumn{4}{|l|}{ Population } \\
\hline Doubling $(\mathrm{h})^{\mathrm{a}}$ & 24.0 & 16.4 & 18.3 \\
\hline $\mathrm{S}+\mathrm{G}_{2} / \mathrm{M}: \mathrm{subG}_{0}^{\mathrm{b}}$ & $1.8 \pm 0.5$ & $9.1 \pm 0.3$ & $9.8 \pm 0.6$ \\
\hline AI colonies $^{c}$ & $0.5 \pm 0.2$ & $27.2 \pm 4.0$ & $28.0 \pm 2.0$ \\
\hline Tumorigenecity $^{\mathrm{d}}$ & $0 / 10$ & $9 / 10$ & $10 / 10$ \\
\hline Tumor latency (weeks) & 24 & $3-5$ & $3-5$ \\
\hline
\end{tabular}

${ }^{a}$ Determined at the exponential growth phase. ${ }^{b}$ Determined by flow cytometry. ${ }^{c}$ Mean anchorage-independent (AI) colony number at day 14 post-seeding of 100 cells/well. ${ }^{\mathrm{d}}$ Determined by the mammary fat pad transplantation assay (21).

on ER/PR/HER-2- MDA-MB-231 cells demonstrate that, relative to the non-tumorigenic 184-B5 cells, the MDA-MB-231 cells exhibited a $53.6 \%$ decrease in the population doubling time, an 8.3-fold decrease in the $\mathrm{S}+\mathrm{G}_{2} / \mathrm{M}$ : $\operatorname{subG}_{0}$ ratio and a 128.7-fold increase in the number of AI colonies. These data are consistent with previously published results (27). Due to the lack of expression of hormone receptors and growth factors, these cells represent a model for triple-negative, chemo-endocrine therapy-resistant clinical breast cancer.

\section{Response to chemo-preventive test compounds}

The prototypic chemopreventive agents were selected as the test compounds based on the evidence for their efficacy in animal models for cancer, and the evidence for their use in the treatment of clinical breast cancer. The data generated from these experiments provide a proof-of-concept for validation of the models as rapid mechanism-based screens to evaluate the preventive/therapeutic efficacy of potential lead compounds.

The synthetic retinoid 4-(hydroxyphenyl) retinamide (4-HPR) has demonstrated efficacy for $\mathrm{ER}^{+}$and $\mathrm{ER}^{-}$clinical breast cancer $(17,19,28)$. The retinoids all-trans retinoic acid (ATRA) and 9-cic retinoic acid (9cis-RA) have already been in clinical use for head and neck cancer $(17,19,29,33)$.

The selective ER modulator, tamoxifen (TAM), has been widely used as a preventive/therapeutic option for the treatment of $\mathrm{ER}^{+}$clinical breast cancer $(3,4,17)$.

The terpenoids carnasol (CSOL), carnosic acid (CA) and ursolic acid (UA) are naturally occurring, bio-active components, functioning as potent COX-2 inhibitors $(17,32,33)$.

The non-fractionated extracts from orange peel (OPE), rosemary (RME) and green tea (GTE) contain mixtures of bio-active flavonoids, terpenoids and polyphenols, respectively, that are known to operate via distinct mechanisms $(15,17,19)$.

The non-fractionated aqueous extracts from select herbal medicinal preparations were selected for use due to their wide use in complementary and alternative medicine for the treatment of breast cancer (17,34-38).

The dose-response experiments with the synthetic retinoid 4-HPR and the selective ER modulator TAM in the MMEC-Ras and MMEC-Myc cell lines demonstrated that the $\mathrm{IC}_{50}$ concentrations for 4-HPR ranged from 2.5 to $2.8 \mu \mathrm{M}$
Table V. Aberrant proliferation and carcinogenic risk in oncogene-transfected human mammary epithelial cells.

\begin{tabular}{lcc}
\hline Biomarker & \multicolumn{2}{c}{ Cell line } \\
\cline { 2 - 3 } & $184-\mathrm{B} 5$ & $184-\mathrm{B} 5 / \mathrm{HER}$ \\
\hline Population & & \\
${\text { Doubling }(\mathrm{h})^{\mathrm{a}}}_{\mathrm{S}+\mathrm{G}_{2} / \mathrm{M}^{\mathrm{s}} \text { subG }_{0}{ }^{\mathrm{b}}}^{\mathrm{AI} \text { colonies }}{ }^{\mathrm{c}}$ & $1.6 \pm 0.3$ & 15 \\
Tumorigenecity $^{\mathrm{d}}$ & $0.3 \pm 0.1$ & $23.0 \pm 2.6$ \\
Tumor latency (weeks) & $0 / 10$ & $10 / 10$ \\
& 24 & $3-5$ \\
\hline
\end{tabular}

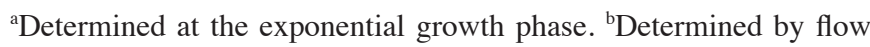
cytometry. ${ }^{\mathrm{c}}$ Mean anchorage-independent (AI) colony number at day 21 post-seeding of 1,000 cells/well. ${ }^{\mathrm{d}}$ Determined by the mammary fat pad transplantation assay.

and that for TAM these ranged from 4.7 to $6.2 \mu \mathrm{M}$. These concentrations in the parental MMEC cells exhibited only 14-18\% growth inhibition, indicating a greater sensitivity of these compounds towards the pre-neoplastically transformed cells (21)

In the 184-B5/HER model for comedo ductal carcinoma in situ (DCIS) the dose-response experiments were conducted with the retinoids 4-HPR, ATRA and 9-cis RA. These agents exhibited an $\mathrm{IC}_{50}$ concentration range of $0.2-2.0 \mu \mathrm{M}$. The experiments with the terpenoids CSOL, CA and UA exhibited a concentration range of 6.2-11.2 $\mu \mathrm{M}$, whereas those with the polyphenol epigallo catechin gallate (EGCG), and flavonoids genistein (GEN) and resveratrol (RES), exhibited an $\mathrm{IC}_{50}$ concentration range of 3.0-8.0 $\mu \mathrm{M}$. These concentrations of retinoids, terpenoids and flavonoids exhibited only $2-20 \%$ inhibition of growth in the parental, non-tumorigenic 184-B5 cells (20,28-33).

In the MDA-MB-231 model for TNBC the dose-response experiments were conducted for non-fractionated extracts OPE, RME and GTE. The $\mathrm{IC}_{50}$ concentrations for these extracts were determined to be $12.5,4.7$ and $9.5 \mu \mathrm{g} / \mathrm{ml}$, respectively. These compounds exhibited only $8-20 \%$ growth inhibition in the parental, non-tumorigenic 184-B5 cells. 
Table VI. Aberrant proliferation and carcinogenic risk in hormone-responsive and hormone-resistant human mammary carcinoma cells.

\begin{tabular}{|c|c|c|c|}
\hline \multirow[t]{2}{*}{ Biomarker } & \multicolumn{3}{|c|}{ Cell line } \\
\hline & 184-B5 & MCF-7 & MDA-MB-231 \\
\hline \multicolumn{4}{|l|}{ Population } \\
\hline Doubling $(\mathrm{h})^{\mathrm{a}}$ & 32.3 & 15.2 & 15.0 \\
\hline $\mathrm{S}+\mathrm{G}_{2} / \mathrm{M}: \mathrm{subG}_{0}^{\mathrm{b}}$ & $1.8 \pm 0.2$ & $10.8 \pm 1.2$ & $16.8 \pm 3.2$ \\
\hline AI colonies ${ }^{c}$ & $0.3 \pm 0.1$ & $30.9 \pm 2.4$ & $38.9 \pm 1.6$ \\
\hline Tumorigenecity $^{\mathrm{d}}$ & $0 / 10$ & $10 / 10$ & $10 / 10$ \\
\hline Latency (weeks) & 24 & $3-5$ & $3-5$ \\
\hline
\end{tabular}

${ }^{a}$ Determined at the exponential growth phase. ${ }^{b}$ Determined by flow cytometry. ${ }^{c}$ Mean anchorage-independent (AI) colony number at day 21 post-seeding of 1,000 cells/well. ${ }^{\mathrm{d}}$ Determined by the mammary fat pad transplantation assay.

In the MCF-7 model for hormone responsive breast cancer, non-fractionated aqueous extracts from several herbal medicinal products were evaluated for their growth inhibitory effects. The test compounds included extracts from Lycium babrabrum bark (LBB), Lyceum babbarum fruit (LBF), Tababuia avellnede (Taheebo, TAH) and Cornus officinalis (CO). The rank order for the efficacy of growth inhibition was determined as $\mathrm{LBB}<\mathrm{CO}<\mathrm{TAH}<\mathrm{LBF}$ (34-38).

\section{Mechanisms for the efficacy of chemo-preventive test compounds}

In the mouse mammary cell culture models for oncogene-initiated mammary carcinogenesis, the synthetic retinoid, 4-HPR, and the selective ER modulator, TAM, induced cell cycle arrest by increasing the $\mathrm{G}_{1}: \mathrm{S}+\mathrm{G}_{2} / \mathrm{M}$ ratio and inducing cell apoptosis. These effects were evident at the pharmacologically achievable concentrations of the test compounds. At the low-dose combinations these agents exhibited synergistic interactions that modulated the two biomarkers. In addition, the combination exhibited a greater efficacy to inhibit AI colony formation (21). These synergistic effects may, in part, be due to distinct mechanisms of action of 4-HPR and TAM.

In the experiments with the human tissue-derived cell culture model for comedo DCIS, the 184-B5/HER (ER $/ \mathrm{PR}^{-} /$ HER-2 ${ }^{+}$) cells were treated with select retinoids (4-HPR, ATRA and 9-cis RA), terpenoids (CSOL, CA and UA) and flavanoids (EGCG, RES and GEN). The data generated from these experiments demonstrated that all of the test compounds, at their respective pharmacologically achievable concentrations, inhibited cell cycle progression, induced cell apoptosis and reduced the number of AI colonies. At the mechanistic level, these compounds inhibited HER-2 signaling, increased the expression of anti-apoptotic $\mathrm{BCl}-2$, decreased the expression of pro-apoptotic $\mathrm{BAX}$ and decreased the transcriptional activity of COX-2 (20,28-33). In addition, the retinoids inhibited cyclin D1 expression and increased the expression of RAR- $\beta$ (29).

In the experiments with the human carcinoma-derived cell culture model for triple-negative breast cancer, MDA-MB-231
(ER $\left./ \mathrm{PR}^{-} / \mathrm{HER}-2^{-}\right)$cells were treated with non-fractionated extracts prepared from OPE, RME and GTE. These extracts, at their respective maximum cytostatic concentrations, inhibited cell cycle progression by inducing $\mathrm{G}_{1}$ arrest, promoting cell apoptosis and reducing the number of AI colonies. The non-fractionated extracts are likely to contain specific, bio-active compounds, such as perillyl alcohol and limonene in OPE, CSOL, CA, UA in RME and EGCG in GTE. These individual bio-active compounds have demonstrated preventive/therapeutic efficacy in numerous preclinical models for breast cancer. Thus, the efficacy of OPE, RME and GTE in MDA-MB-231 cells may, in part, be due to the presence of specific bio-active components of the non-fractionated extracts $(32,33)$.

In the experiments using the human carcinoma-derived model for chemo-endocrine therapy-responsive breast cancer, MCF-7 (ER $\left./ \mathrm{PR}^{+} / \mathrm{HER}-2^{-}\right)$cells were treated with non-fractionated aqueous extracts prepared from numerous herbal medicinal products that are in use for breast cancer in complementary and alternative medicine. The extract prepared from LBF (at its maximum cytostatic concentration) reduces the number of AI colonies. At the mechanistic level, LBF treatment modulates the cellular metabolism of $17 \beta$-estradiol $\left(\mathrm{E}_{2}\right)$ to increase the formation of the anti-proliferative metabolite $2-\mathrm{OHE}_{1}$ and to accelerate the conversion of the pro-mitogenic $16 \alpha-\mathrm{OHE}_{1}$ to mitogenically inert $\mathrm{E}_{3}(34)$. The extract prepared from LBB inhibits AI colony formation, predominantly due to the increased production of $2-\mathrm{OHE}_{1}(35)$. The extract prepared from the bark of Tabebuia avellende inhibits cell cycle progression, induces cell apoptosis and modulates the global gene expression profiles of genes specific for cell cycle regulation, cell apoptosis, ER and the xeno-biotic metabolism (36). The extract prepared from the fruit of Fructus cornii, also known as Cornus officinalis, exhibits growth inhibitory effects in MCF-7 cells, predominantly via modulation of the cell metabolism of $\mathrm{E}_{2}(37,38)$. Overall, the data generated from the studies on MCF-7 cells provide proof-of-principle evidence for the therapeutic efficacy of herbal medicinal products. However, specific bio-active components, susceptible biochemical pathways and molecular targets for the efficacy of herbal medicinal products remain to be identified. 


\section{Conclusions and future directions}

Conventional animal models for breast cancer include chemical carcinogen-induced mammary cancer model and genetically predisposed models, where the targeted expression of selected oncogenes drive the carcinogenic process. Consistent with these models, the mouse mammary epithelial cell culture models exhibited induction of the multi-step carcinogenic process in response to treatment with the chemical carcinogen DMBA and also in response to the targeted overexpression of Ras and Myc oncogenes (20-25). In 184-B5 cells, DMBA and BP, and in 184-B5/HER cells, targeted overexpression of the HER-2 oncogene induced carcinogenic transformation $(20,25)$. In all these preclinical cell culture models the multi-step process of carcinogenic transformation is associated with a loss of homeostatic growth control and gain of carcinogenic risk, as evidenced by aberrant proliferation, accelerated cell cycle progression, downregulation of cell apoptosis, enhancement of AI in vitro and tumor development upon in vivo transplantation (20-25). Thus, these models provide valuable mechanism-based approaches, complementing the existing in vivo models of transgenic mice and of athymic mice xeno-transplanted with human mammary carcinoma-derived cells, respectively.

The investigations conducted to validate the developed models as rapid mechanistic approaches and to evaluate the efficacy of preventive/therapeutic lead compounds utilized prototypical pharmacological agents as test compounds. Selective ER modulator TAM, as well as the retinoids 4-HPR, ATRA and 9cis-RA, are already in clinical use for the cancer of various organ sites and function via distinct mechanisms. These test compounds modulated aberrant cell cycle progression and induced cell apoptosis, thereby re-establishing homeostatic growth control, and decreasing the number of AI colonies, thus reducing the carcinogenic risk $(21,28,29)$. Comparative studies with non-tumorigenic MMEC and 184-B5, and pre-neoplastic MMEC-Ras, MMEC-Myc and 184-B5/HER cells revealed a greater sensitivity of pre-neoplastic cells to the test compounds. These observations validate an approach to evaluate the sensitivity and specificity of efficacious lead compounds.

Long-term use of conventional chemotherapeutic drugs is frequently associated with acquired tumor resistance and/ or adverse systemic toxicity, leading to compromised patient compliance $(3,4)$. Bio-active components, present in natural phytochemicals, generally exhibit acceptable toxicity profiles. Mechanistic interaction of natural phytochemicals with conventional chemotherapeutic agents may lead to enhanced efficacy and reduced therapy-associated toxicity $(15,17-19)$. This concept represents a testable alternative.

The studies on human mammary carcinoma-derived models utilized numerous non-fractionated extracts from naturally occurring compounds and herbal medicinal preparations. These test compounds, operating via distinct mechanisms, exhibited anti-proliferative and/or pro-apoptotic effects (35-38).

Investigations focusing on cancer-initiating stem cells have provided convincing evidence to support the concept that this sub-population may largely be responsible for acquired tumor resistance to chemotherapy and the resultant ineffectiveness of conventional treatment options (39). The isolation and characterization of putative cancer-initiating stem cells provides a novel approach to identify efficacious agents for the targeted therapy of chemo-endocrine therapy-responsive and -resistant clinical breast cancer $(40,41)$.

Numerous recent publications have identified putative cancer stem cell phenotypes by quantifying the expression of CD44/CD24, ALDH-1 and ABCG2 cellular proteins, and NANOG, OCT-4, SOX-2 and c-Myc nuclear transcription factors. Modulations in the expression of these molecules may, in part, be responsible for the observed properties of self-renewal, chemotherapeutic drug resistance and accelerated tumorigenic potential that represent specific characteristics of cancer-initiating stem cell populations (42-47).

Aberrant proliferation, downregulated cell apoptosis and specific response to chemo-therapeutic agents in the cell culture model for comedo DCIS have provided conceptual and mechanistic leads supporting the presence of putative cancer-initiating stem cells in this model. Our recent studies on the ER ${ }^{-} / \mathrm{PR}^{-} / \mathrm{HER}^{2}{ }^{+}$184-B5/HER model for comedo DCIS have demonstrated that retinoid-resistant phenotypes may be isolated from the parental cells that survive a high-dose treatment of 4-HPR and ATRA. These drug-resistant phenotypes exhibit persistent hyper-proliferation in the presence of 4-HPR and ATRA and exhibit enhanced AI growth (48). Current studies are focused on the extensive mechanism-based molecular characterization of retinoid-resistant cancer-initiating stem cells, using previously documented relevant cell proteins, nuclear transcriptional factors and quantifiable carcinogenic risk as end-point biomarkers. The data generated following these experimental strategies are expected to establish the utility of cancer-initiating stem cell-derived models for stem cell-targeted therapeutic approaches for clinical breast cancer.

\section{Acknowledgements}

The authors would like to acknowledge the outstanding efforts of all post-doctoral research associates, surgical fellows and research technicians for their participation in the research program. This research program has been funded in the past by the US National Cancer Institute Grants (CA-44741, CA-29502), and Contract (CN-75029-63), the US Department of Defense Breast Cancer Research Program Grant (DAMD1794-J-4208) and by philanthropic support to the Strang Cancer Prevention Center.

\section{References}

1. Siegel R, Ward E, Brawley O and Jemal A: Cancer statistics, 2011: the impact of eliminating socioeconomic and racial disparities on premature cancer deaths. CA Cancer J Clin 61: 212-236, 2011.

2. Martin AM and Weber BL: Genetic and hormonal risk factors in breast cancer. J Natl Cancer Inst 92: 1126-1135, 2000.

3. Lippman ME: Efforts to combine endocrine and chemotherapy in the management of breast cancer: do two and two equal three? Breast Cancer Res Treat 3: 117-127, 1983.

4. Early Breast Cancer Trialists' Collaborative Group (EBCTCG): Effects of chemotherapy and hormonal therapy for early breast cancer on recurrence and 15-year survival: an overview of the randomised trials. Lancet 365: 1687-1717, 2005.

5. Sørlie T, Perou CM, Tibshirany R, et al: Gene expression patterns of breast carcinomas distinguish tumor subclasses with clinical implications. Proc Natl Acad Sci USA 98: 10869-10874, 2001.

6. National Comprehensive Cancer Network: NCCN clinical practice guidelines in oncology: Breast Cancer 1: 2010 http://www.nccn.org.

7. Perou CM, Sørlie T, Eisen MB, et al: Molecular portraits of human breast tumors. Nature 406: 747-752, 2000. 
8. Neve RM, Chin K, Fridlyand J, et al: A collection of breast cancer cell lines for the study of functionally distinct cancer subtypes Cancer Cell 10: 515-527, 2006

9. Ontilo AA, Engel JM, Greenlee RT and Mukesh BN: Breast cancer subtypes based on ER/PR and Her2 expression: comparison of clinicopathologic features and survival. Clin Med Res 7: 4-13, 2009.

10. Salmon DJ, Leyland-Jones, B, Shak S, et al: Use of chemotherapy plus a monoclonal antibody against HER 2 for metastatic breast cancer that overexpresses HER2. N Eng J Med 344: 783-792, 2001

11. Piccart-Gebhart MJ, Procter M, Leyland-Jones B, et al: Trastuzumab after adjuvant chemotherapy in HER2-positive breast cancer. N Eng J Med 353: 1659-1672, 2005.

12. Romond EH, Perez EA, Bryant J, et al: Trastuzumab plus adjuvant chemotherapy for operable HER2-positive breast cancer. N Eng J Med 353: 1673-1684, 2005.

13. Baselga J and Swain SM: Novel anti-cancer targets: revisiting ERBB2 and discovering ERBB3. Nat Rev Cancer 9: 463-475, 2009.

14. Cleator S, Heller W and Coombes RC: Triple-negative breast cancer: therapeutic options. Lancet Oncol 8: 235-244, 2007.

15. Sarkar FH and $\mathrm{Li}$ Y: Using chemopreventive agents to enhance the efficacy of cancer therapy. Cancer Res 66: 3347-3350, 2006.

16. Rock E and DeMichele A: Nutritional approaches to late toxicities of adjuvant chemotherapy in breast cancer survivors. J Nutr 133: S3785-S3793, 2003.

17. Shureiqi I, Reddy P and Brenner DE: Chemoprevention: general perspective. Crit Rev Oncol 33: 157-167, 2000

18. Bradlow HL, Telang NT, Sepkovic DW and Osborne MP Phytochemicals as modulators of cancer risk. Adv Exp Med Biol 207-221, 1999.

19. Abbruzzese JL and Lippman SM: The convergence of cancer prevention and therapy in early-phase clinical drug development. Cancer Cell 6: 321-326, 2004.

20. Katdare M, Osborne MP and Telang N: Novel cell culture models for prevention of human breast cancer (Review). Int J Oncol 22: 509-515, 2003.

21. Telang $\mathrm{N}$ and Katdare $\mathrm{M}$ : Preclinical in vitro models from genetically engineered mice for breast and colon cancer (Review) Oncol Rep 25: 1195-1201, 2011.

22. Garg A, Osborne M, Gupta R, et al: Expression of biomarkers for transformation in 7,12-dimethylbenz[a]anthracene-treated mammary epithelial-cells. Int J Oncol 3: 185-189, 1993.

23. Telang NT, Narayanan R, Bradlow HL and Osborne MP Coordinated expression of intermediate biomarkers for tumorigenic transformation in RAS-transfected mouse mammary epithelial cells. Breast Cancer Res Treat 18: 155-163, 1991.

24. Telang NT, Osborne MP, Sweterlitsch LA, et al: Neoplastic transformation of mouse mammary epithelial cells by deregulated myc expression. Cell Regul: 863-872, 1990.

25. Katdare M, Osborne MP and Telang NT: Inhibition of aberrant proliferation and induction of apoptosis in pre-neoplastic human mammary epithelial cells by natural phytochemicals. Oncol Rep 5: 311-315, 1998.

26. Suto A, Bradlow HL, Kubota T, et al: Alteration in proliferative and endocrine responsiveness of human mammary carcinoma cells by prototypic tumor-suppressing agents. Steroids 58 215-219, 1993.

27. Telang NT, Katdare M, Bradlow HL, Osborne MP and Fishman J: Inhibition of proliferation and modulation of estradiol metabolism: novel mechanisms for breast cancer prevention by the phytochemical indole-3-carbinol. Proc Soc Exp Biol Med 216: 246-252, 1997.

28. Jinno H, Steiner MG, Mehta RG, et al: Inhibition of aberrant proliferation and induction of apoptosis in HER-2/neu oncogene transformed human mammary epithelial cells by N-(4-hydroxyphenyl)retinamide. Carcinogenesis 20: 229-236, 1999.

29. Jinno H, Steiner MG, Nason-Burchenal K, et al: Preventive efficacy of receptor class selective retinoids on HER-2/neu oncogene expressing preneoplastic human mammary epithelial cells. Int J Oncol 21: 127-134, 2002.
30. Subbaramaiah K, Chung WJ, Micheluart P, et al: Resveratrol inhibits cyclooxygenase- 2 transcription and activity in phorbol ester-treated human mammary epithelial cells. J Biol Chem 273: 21875-21882, 1998

31. Katdare M, Osborne M and Telang NT: Soy isoflavone genistein modulates cell cycle progression and induces apoptosis in HER-2/neu oncogene expressing human breast epithelial cells Int J Oncol 21: 809-815, 2002.

32. Subbaramaiah K, Michaluart P, Sporn MB and Dannenberg AJ: Ursolic acid inhibits cyclooxygenase-2 transcription in human mammary epithelial cells. Cancer Res 60: 2399-2404, 2000.

33. Subbaramaiah K, Cole PA and Dannenberg AJ: Retinoids and carnosol suppress cyclooxygenase-2 transcription by CREBbinding protein/p300-dependent and -independent mechanisms. Cancer Res 62: 2522-2530, 2002.

34. Li G, Sepkovic DW, Bradlow HL, Telang NT and Wong GY: Lycium barbarum inhibits growth of estrogen receptor positive human breast cancer cells by favorably altering estradiol metabolism. Nutr Cancer 61: 408-414, 2009.

35. Telang NT, Sepkovic DW, Bradlow HL and Wong GY: Comparative preventive efficacy of aqueous extracts from lycium barbarum bark and fruit on estrogen receptor positive human mammary carcinoma MCF-7 cells. Cancer Res 71: 379S, 2011.

36. Mukherjee B, Telang N and Wong GY: Growth inhibition of estrogen receptor positive human breast cancer cells by Taheebo from the inner bark of Tebebuia avellandae tree. Int J Mol Med 24: 253-260, 2009.

37. Telang NT, Sepkovic DW, Bradlow HL and Wong GYC: Chinese herb Fructus cornii exhibits preventive efficacy in a cell culture model for hormone responsive breast cancer. Cancer Res 70 : $152 \mathrm{~S}, 2010$

38. Telang NT, Li G, Sepkovic DW, et al: Anti-proliferative effects of Chinese herb Cornus officinalis in a cell culture model for estrogen receptor-positive clinical breast cancer. Mol Med Report 5: 22-28, 2012.

39. Moore KA and Lemishka IR: Stem cells and their niches. Science 311: 1880-1885, 2006.

40. Dean M, Fojo T and Bates S: Tumor stem cells and drug resistance. Nat Rev Cancer 5: 275-284, 2005

41. Al-Hajj M, Wicha MS, Benito-Hernandez A, et al: Prospective identification of tumorigenic breast cancer cells. Proc Natl Acad Sci USA 100: 3983-3988, 2003.

42. Stingl J and Caldas C: Molecular heterogeneity of breast carcinomas and the cancer stem cell hypothesis. Nat Rev Cancer 7: 791-799, 2007

43. Patel SA, Ndabahaliye A, Lim PK, et al: Challenges in the development of future treatments for breast cancer stem cells. Breast Cancer (London) 2: 1-11, 2010.

44. Van Phuc P, Nhan PL, Nhung TH, et al: Downregulation of $\mathrm{CD} 44$ reduces doxorubicin resistance of $\mathrm{CD} 44 \mathrm{CD} 24$ breast cancer cells. Onco Targets Ther 4: 71-78, 2011.

45. Tanei T, Morimoto K, Shimazu K, et al: Association of breast cancer stem cells identified by aldehyde dehydrogenase 1 expression with resistance to sequential paclitaxel and epirubicin-based chemotherapy for breast cancers. Clin Cancer Res 15: 4234-4241, 2009.

46. Asiedu MK, Ingle JN, Behrens MD, et al: TGF- $\beta / T N F-\alpha$-mediated epithelial-mesenchymal transition generates breast cancer stem cells with a claudin-low phenotype. Cancer Res 71: 4707-4719, 2011.

47. Zhang F, Song C, Ma Y, et al: Effect of fibroblasts on breast cancer cell mammosphere formation and regulation of stem cellrelated gene expression. Int J Mol Med 28: 365-371, 2011.

48. Telang $\mathrm{N}$ and Katdare M: Isogenic human breast epithelial cell lines: Model for cancer initiating stem cells. Int J Mol Med 28: S19, 2011 